\title{
ALGEBRAIC COMPACTNESS OF ULTRAPOWERS AND REPRESENTATION TYPE
}

\author{
C. U. Jensen AND B. ZimmermanN-Huisgen
}

It is shown that for certain clases of Artin algebras infinite representation type is equivalent to the existence of a module $M$ all of whose ultrapowers $M^{\mathbb{N}} / \mathscr{F}$ fail to be algebraically compact.

1. Introduction. As is well known, an Artin algebra $R$ is of finite representation type, i.e., $R$ admits only finitely many isomorphism classes of indecomposable right or left modules, if and only if $R$ has right pure global dimension zero (combine [1] with [5] or [13]). The latter condition means that all right $R$-modules are pure-injective or, equivalently, algebraically compact; in particular, it of course entails algebraic compactness of all non-trivial countable ultraproducts of right $R$-modules. (Call an ultraproduct countable if it extends over a countable index set, non-trivial if the corresponding ultrafilter is non-principal.)

Let us leave this "extreme" case. How do the non-trivial countable ultraproducts of $R$-modules reflect higher pure global dimensions of the ground ring?

The situation where $R$ is countable is exceptional on that score: namely, non-trivial countable ultraproducts of $R$-modules are always algebraically compact in that case (a short proof can be found in the appendix; see also [4, Theorem 42.1] and [6, Remarque 7.12]). On the other hand, if $R$ is an uncountable Artin algebra of a pure global dimension exceeding 0 , then "usually" there exist non-trivial countable ultraproducts of finitely generated modules which fail to be algebraically compact (see [8] for precise statements). Interestingly, this conclusion can be translated back to a stronger condition on the pure global dimension of $R$; namely, it rules out the values 0 and 1 .

The picture for ultrapowers is quite different in that arbitrary ultrapowers of finitely generated modules over an Artin algebra are algebraically compact (see the appendix for details). Any case of failure of algebraic compactness for a non-trivial countable ultrapower $M^{\mathbb{N}} / \mathscr{F}$ thus involves a non-finitely generated module $M$ over an uncountable Artin algebra of infinite type. In fact, for Artin algebras $R$ 
which are either

(A) commutative, with all local direct summands uncountable or

(B) group algebras over uncountable fields

we show the equivalence of the following two statements:

(1) $R$ is not of finite representation type.

(2) There exists an $\aleph_{1}$-generated $R$-module $M$ such that for all filters $\mathscr{F}$ on $\mathbb{N}$ the reduced power $M^{\mathbb{N}} / \mathscr{F}$ fails to be algebraically compact. Individual steps of our arguments, e.g. a computation of pure-injective envelopes and some general observations concerning ultrapowers of direct sums, should be of independent interest and applicable in different contexts.

In an appendix we assemble a few staples on ultraproducts, the majority of which are known. In particular, their specializations to $R=\mathbb{Z}$ have been widely used and can be found in [4]. Even though the more general statements are easy extensions of the latter, we include them for convenient reference.

Terminology and a few facts in the background. Recall that a right $R$-module $M$ is called algebraically compact if every finitely solvable system of linear equations

$$
\sum_{i \in I} X_{i} r_{i j}=m_{j} \quad(j \in J),
$$

where $\left(r_{i j}\right)_{i \in I, j \in J}$ is a column-finite matrix over $R$ and all $m_{j}$ belong to $M$, has a global solution in $M^{I}$. According to [10, Theorem 2] this property is equivalent to pure-injectivity of $M$ (i.e. injectivity with respect to pure inclusions). A pure-injective envelope of $M$ is a pure inclusion $M \subseteq E$ such that $E$ is pure-injective and does not contain a non-zero submodule $N$ with $N \cap M=0$ and $M \rightarrow E / N$ pure.

Finally, given a family $\left(M_{i}\right)_{i \in I}$ of modules and a filter $\mathscr{F}$ on $I$, consider the submodule $U(\mathscr{F})$ of the direct product $\prod_{i \in I} M_{i}$ consisting of those elements $\left(x_{i}\right)_{i \in I}$ for which $\left\{i \in I \mid x_{i}=0\right\}$ belongs to $\mathscr{F}$. The quotient $\prod_{i \in I} M_{i} / U(\mathscr{F})$ is abbreviated by $\prod_{i \in I} M_{i} / \mathscr{F}$ and called a reduced product (modulo $\mathscr{F}$ ). If $M_{i} \cong M$ for all $i \in I$, we simplify the notation further to $M^{I} / \mathscr{F}$ and call $M^{I} / \mathscr{F}$ a reduced power. In case $\mathscr{F}$ is an ultrafilter on $I$, we say that $\prod_{i \in I} M_{i} / \mathscr{F}$ is an ultraproduct resp. $M^{I} / \mathscr{F}$ is an ultrapower; we use the qualifier non-trivial for ultraproducts resp. ultrapowers to indicate that the corresponding ultrafilter is non-principal. The residue class of an element $m=\left(m_{i}\right)_{i \in I} \in \prod_{i \in I} M_{i}$ in $\prod_{i \in I} M_{i} / \mathscr{F}$ we denote by $[m]$ or $\left[m_{i}\right]_{i \in I}$. 
2. Ultrapowers over commutative artinian rings. Throughout this section, $R$ denotes a commutative artinian ring with Jacobson radical $J$. The present goal is to prove the following

THEOREM 1. Suppose that $R$ is a commutative artinian ring all of whose local direct summands are uncountable. Then the following statements are equivalent:

(1) $R$ is not a principal ideal ring.

(2) There exists an $\aleph_{1}$-generated $R$-module $M$ such that, for each filter $\mathscr{F}$ on $\mathbb{N}$, the reduced power $M^{\mathbb{N}} / \mathscr{F}$ is not algebraically compact.

(3) If $M$ is the direct sum $\bigoplus R / I$, where I runs through all ideals between $J$ and $J^{2}$, then $M^{\mathbb{N}} / \mathscr{F}$ is not algebraically compact for any filter $\mathscr{F}$ on $\mathbb{N}$.

The commutative rings of finite representation type being precisely the artinian principal ideal rings, the implications " $(2) \Rightarrow(1)$ " and "(3) $\Rightarrow(1)$ " follow immediately from our introductory remarks. The road for a proof of "(1) $\Rightarrow(2)$ and (3)" will be smoothed through a series of lemmas. We emphasize the fact that our argument does not use the continuum hypothesis (by adopting it, we could significantly simplify our proof).

Since we will not lose generality on that account, we will assume for the rest of this section that $R$ is local.

LEMMA 2 (Warfield [11]). If $R$ is uncountable and not a principal ideal ring, then $R / J$ is uncountable, and there exists an ideal I with $J^{2} \subseteq I \subset J$ such that $J / I$ has $R / J$-dimension 2 .

Proof. $R / J$ is uncountable, since each of the quotients $J^{n} / J^{n+1}$ is finite dimensional over $R / J$, and

$$
|R|=\prod_{n=0}^{l}\left|J^{n} / J^{n+1}\right|
$$

where $l$ is the Loewy length of $R$. By [11, pp. 451/52], $R$ contains an ideal $I$ as required.

Given any quotient $R / I$ of $R$, every non-algebraically compact $R / I$ module is, a fortiori, not algebraically compact as an $R$-module. In proving Theorem 1 we may thus, in view of Lemma 2, restrict ourselves to dealing with the situation where $J^{2}=0$ and the dimension of $J$ over $R / J$ is 2 . Moreover, we will assume from now on that $R$, 
and hence also $R / J$, is uncountable. Let $K \subseteq R \backslash J$ be a set of representatives for $R / J$, i.e. $\{\alpha+J: \alpha \in K\}=R / J$ and $\alpha+J \neq \beta+J$ for $\alpha, \beta \in K, \alpha \neq \beta$, and let $k \subseteq K$ be a subset of cardinality $\aleph_{1}$. Next pick an $R / J$-basis $x, y$ for $J$, and set $M_{\alpha}=R /(x-y \alpha)$ for $\alpha \in k$. Note that, for each $\alpha$, the $R$-module $M_{\alpha}$ has length 2 and radical $M_{\alpha} J=\bar{y} R$, where $\bar{y}$ denotes the residue class of $y$ in $M_{\alpha}$. (For notational convenience, we will denote all residue classes by bars; it should be clear from the context which quotient we will be considering.) In the sequel, we will inspect the module

$$
M=\bigoplus_{\alpha \in k} M_{\alpha}
$$

under a magnifying glass.

LEMMA 3. The canonical inclusion $M=\bigoplus_{\alpha \in k} M_{\alpha} \subseteq \prod_{\alpha \in k} M_{\alpha}$ is a pure-injective envelope of $M$.

Proof. Set $E=\prod_{\alpha \in k} M_{\alpha}$. Since each $M_{\alpha}$ is algebraically compact over $R$ (compare e.g. [14]), the same is true for $E$. Thus $E$ contains as a direct summand a pure-injective envelope $B$ of $M$, say $E=B \oplus C$.

In order to see that $E=B$, we will first verify that $E J \subseteq B$. Start by observing that each element of $E J$ has the form ${\overline{(y \beta(\alpha))_{\alpha \in k}}}_{i} \in$ $\prod_{\alpha \in k} M_{\alpha}$ for some family $(\beta(\alpha))_{\alpha \in k}$ of elements of $K$. Then, fixing such a family $(\beta(\alpha))_{\alpha \in k}$, consider the following system of equations in the unknowns $\xi$ and $\eta_{\alpha}, \alpha \in k$, with right-hand sides in $M$ :

$$
\xi+\eta_{\alpha}(x-y \alpha)=e_{\alpha} y \beta(\alpha), \quad \alpha \in k,
$$

where $e_{\alpha} \in M$ is the element carrying $\overline{1}$ in the $\alpha$ th slot and 0 elsewhere. This system is finitely solvable in $M$ : In fact, given a finite subset $F \subseteq$ $k$, the elements $\xi=\sum_{\varphi \in F} e_{\varphi} y \beta(\varphi)$ and $\eta_{\alpha}=\sum_{\varphi \in F \backslash\{\alpha\}} e_{\varphi} \beta(\varphi) \gamma(\alpha-\varphi)$, where $\gamma(\alpha-\varphi) \in K$ is such that $(\alpha-\varphi) \cdot \gamma(\alpha-\varphi) \equiv 1(\bmod J)$, constitute a solution of the finite subsystem indexed by $F$. Since $B$ is algebraically compact and $M \subseteq B$, the global system has a solution $\xi$, $\left(\eta_{\alpha}\right)_{\alpha \in k}$ in $B$. But the nature of our system implies $\xi=\overline{(y \beta(\alpha))}_{\alpha \in k}$, whence $E J \subseteq B$.

Because $E=B \oplus C$, we deduce $C J=0$, meaning that

$$
C \subseteq \prod_{\alpha \in k}\left(M_{\alpha} J\right)=E J \subseteq B
$$

Thus $C=0$. 
The following construction and lemma apply not only to our present setting, but are clearly independent of the nature of the $M_{\alpha}$ (in fact, one can substitute arbitrary right modules for the $M_{\alpha}$ ).

Given $M=\bigoplus_{\alpha \in k} M_{\alpha}$ as introduced above and a filter $\mathscr{F}$ on $\mathbb{N}$, consider the canonical map

$$
\begin{gathered}
\Phi: M^{\mathbb{N}} / \mathscr{F} \rightarrow \prod_{\alpha \in k}\left(M_{\alpha}^{\mathbb{N}} / \mathscr{F}\right), \\
{\left[\left(m_{\alpha n}\right)_{\alpha \in k}\right]_{n \in \mathbb{N}} \rightarrow\left(\left[m_{\alpha n}\right]_{n \in \mathbb{N}}\right)_{\alpha \in k}}
\end{gathered}
$$

and the "diagonal map"

$$
\begin{aligned}
\Delta: M & \rightarrow M^{\mathbb{N}} / \mathscr{F}, \\
m & \rightarrow[m]_{n \in \mathbb{N}} .
\end{aligned}
$$

Moreover, set $U_{\alpha}=M_{\alpha}^{\mathbb{N}} / \mathscr{F}$, and denote the submodule of $\prod_{\alpha \in k} U_{\alpha}$ consisting of the elements of countable support by $\widehat{\prod}_{\alpha \in k} U_{\alpha}$.

LEMMA 4.

(a) The image of $\Phi$ is contained in $\widehat{\prod}_{\alpha \in k} U_{\alpha}$.

(b) $\Delta(M) \cap \operatorname{Ker}(\Phi)=0$.

(c) The inclusion $\Delta(M) \subseteq M^{\mathbb{N}} / \mathscr{F}$ is pure and so is the canonical embedding $\Delta(M) \rightarrow\left(M^{\mathbb{N}} / \mathscr{F}\right) / \operatorname{Ker}(\Phi)$.

Proof. Parts (a), (b) and the first statement of (c) are obvious. We will sketch an approach to the second part of (c) which is less messy than the straightforward one. Consider the following diagram

$$
\begin{array}{cll}
M=\bigoplus_{\alpha \in k} M_{\alpha} & \stackrel{\bigoplus^{\Delta_{n}}}{\longrightarrow} & \bigoplus_{\alpha \in k}\left(M_{\alpha}^{\mathbb{N}} / \mathscr{F}\right) \subseteq \prod_{\alpha \in k}\left(M_{\alpha}^{\mathbf{N}} / \mathscr{F}\right) \\
\Delta(M) & \stackrel{\text { can }}{\longrightarrow} & \left(M^{\mathbb{N}} / \mathscr{F}\right) / \operatorname{Ker}(\Phi)
\end{array}
$$

where $\Delta_{\alpha}$ is the (pure) diagonal embedding $M_{\alpha} \rightarrow M_{\alpha}^{\mathbb{N}} / \mathscr{F}, \bar{\Phi}$ is the monomorphism induced by $\Phi$ and can is the inclusion whose purity is at stake. Since both of the inclusions in the top row are pure, so is the composite, and the purity of can follows via a straightforward computation.

The next lemma is a variation of a variation of a motif of Chase (see [2] for the original motif, [3] for a primary variation). Even though the argument is closely modeled after that given in [13], we include it for the sake of completeness. The functors in which we will later be interested are of the form $\operatorname{Mod}-R \rightarrow \mathrm{Ab}, X \rightarrow \bigcap_{l \in L}\left(X \mathfrak{a}_{l}\right)$, where 
$\left(\mathfrak{a}_{l}\right)_{l \in L}$ is a family of ideals of $R$; their only crucial property in this context is the fact that they are subfunctors of the forgetful functor Mod $-R \rightarrow$ Mod $-\mathbb{Z}$ commuting with direct products. (A subfunctor of the forgetful functor is a functor $F: \operatorname{Mod}-R \rightarrow \operatorname{Mod}-\mathbb{Z}$ such that $F X$ is a subgroup of $X$ for each $R$-module $X$, and $f(F X) \subseteq F Y$ whenever $f: X \rightarrow Y$ is an $R$-module homomorphism.) For a pair of such functors, $F_{1}$ and $F_{2}$ say, we write $F_{1} \supseteq F_{2}$ if $F_{1} X \supseteq F_{2} X$ for all $R$-modules $X$.

Given $R$-modules $B_{l}, l \in L$, we will again denote by $\hat{\prod}_{l \in L} B_{l}$ the submodule of the direct product consisting of all elements of countable support. The first uncountable ordinal number we will write as $\Omega$.

LeMma 5. Let $\left(A_{i}\right)_{i \in I}$ and $\left(B_{l}\right)_{l \in L}$ be two families of $R$-modules, where $|I| \geq \aleph_{1}$, and let

$$
f: \prod_{i \in I} A_{i} \rightarrow \widehat{\prod_{l \in L}} B_{l}
$$

be a homomorphism. Moreover, suppose that $\left(F_{\tau}\right)_{\tau<\Omega}$ is a chain of subfunctors of the forgetful functor Mod $-R \rightarrow \operatorname{Mod}-\mathbb{Z}$ which commute with direct products, such that $F_{\sigma} \supseteq F_{\tau}$ for $\sigma<\tau<\Omega$. Then there exists an ordinal number $\rho<\Omega$, an uncountable subset $\widetilde{I} \subseteq I$ and $a$ countable subset $\widetilde{L} \subseteq L$ such that

$$
f\left(F_{\rho} \prod_{i \in \widetilde{I}} A_{i}\right) \subseteq \prod_{l \in \widetilde{L}} B_{l}+\bigcap_{\tau<\Omega} F_{\tau}\left(\prod_{l \in L} B_{l}\right) .
$$

Proof. Start by observing that the functors $F_{\tau}$ automatically commute with "thinned" direct products $\widehat{\Pi}$.

W.l.o.g. we may assume

$$
I=\{\tau \mid \tau \text { is an ordinal number with } \tau<\Omega\} .
$$

Moreover, since $\prod_{i \in I} A_{i} \cong \prod_{i \in I} \bar{A}_{i}$, where each $\bar{A}_{i}$ is an uncountable direct product of $A_{i}$ 's, it is clearly enough to prove the existence of an ordinal number $\rho<\Omega$ and a countable subset $\widetilde{L}$ of $L$ such that

$$
f\left(F_{\rho} A_{\rho}\right) \subseteq \prod_{l \in \widetilde{L}} B_{l}+\bigcap_{\tau<\Omega} F_{\tau}\left(\widehat{\prod}_{l \in L} B_{l}\right) .
$$

Assume the contrary. Our goal is to construct an element $x \in \prod_{\tau<\Omega} A_{\tau}$ such that $f(x)$ has uncountable support (which is incompatible with $\left.f(x) \in \widehat{\prod}_{l \in L} B_{l}\right)$. 
Pick any $\tau_{0}<\Omega$. If we denote by $p_{j}$ the $j$ th canonical projection $\widehat{\prod}_{l \in L} B_{l} \rightarrow B_{j}$, the fact that

$$
f\left(F_{\tau_{0}} A_{\tau_{0}}\right) \nsubseteq \bigcap_{\alpha<\Omega} F_{\alpha}\left(\widehat{\prod} B_{l \in L}\right)
$$

entails the existence of an ordinal $\tau_{1}$ with $\tau_{0}<\tau_{1}<\Omega$, an element $x_{0} \in F_{\tau_{0}} A_{\tau_{0}}$ and an element $l_{0} \in L$ such that $p_{l_{0}} f\left(x_{0}\right) \in F_{\tau_{0}} B_{l_{0}} \backslash F_{\tau_{1}} B_{l_{0}}$.

Let us set out from the situation where, for some $\rho<\Omega$, we already are in the possession of ordinal numbers $\tau_{\nu}$ and $\tau_{\nu+1}<\Omega$ for all $\nu<\rho$, of elements $x_{\nu} \in F_{\tau_{\nu}} A_{\tau_{\nu}}$, and elements $l_{\nu} \in L$ such that

(1) $\tau_{\mu}<\tau_{\nu}$ for $\mu<\nu$,

(2) $l_{\mu} \neq l_{\nu}$ for $\mu \neq \nu$,

(3) $p_{l_{\nu}} f\left(x_{\nu}\right) \notin F_{\tau_{\nu+1}} B_{l_{\nu}}$ for $\nu<\rho$,

(4) $p_{l_{\nu}} f\left(\sum_{\mu<\nu} x_{\mu}\right)=0$ for $\nu<\rho$.

(Observe that $\sum_{\mu<\nu} x_{\mu} \in \prod_{\tau<\Omega} A_{\tau}$ is well defined, because the $\tau_{\mu}$ are distinct.)

Suppose first that $\rho$ is a limit ordinal. In this case we need to find $\tau_{\rho}, \tau_{\rho+1}, x_{\rho}$ and $l_{\rho}$ subject to the above conditions (1)-(4). Since $\sup _{\nu<\rho} \tau_{\nu+1}=\sup _{\nu<\rho} \tau_{\nu}$ is countable we can choose $\tau_{\rho}$ with $\sup _{\nu<\rho} \tau_{\nu}$ $<\tau_{\rho}<\Omega$. Clearly, $a=\sum_{\nu<\rho} x_{\nu} \in \sum_{\tau<\Omega} A_{\tau}$ is well defined, and since the support of $f(a)$,

$$
\widetilde{L}=\left\{l \in L \mid p_{l} f(a) \neq 0\right\},
$$

is countable, our assumption says

$$
f\left(F_{\tau_{\rho}} A_{\tau_{\rho}}\right) \nsubseteq \prod_{l \in \widetilde{L}} B_{l}+\bigcap_{\alpha<\Omega} F_{\alpha}\left(\widehat{\prod} B_{l \in L} B_{l}\right) .
$$

Pick an element $x_{\rho} \in F_{\tau_{\rho}} A_{\tau_{\rho}}$, an ordinal $\tau_{\rho+1}$ with $\tau_{\rho}<\tau_{\rho+1}<\Omega$ and an element $l_{\rho} \in L \backslash \widetilde{L}$ such that

$$
P_{l_{\rho}} f\left(x_{\rho}\right) \notin F_{\tau_{\rho+1}} B_{l_{\rho}}
$$

(keep in mind that $F_{\mu} X \supseteq F_{\nu} X$ for any $R$-module $X$, whenever $\nu<\mu$ ). By definition of $\widetilde{L}$ we have $P_{l_{\rho}} f\left(\sum_{\nu<\rho} x_{\nu}\right)=0$ and, in view of the inclusion $\left\{l_{\nu}: \nu<\rho\right\} \subseteq \widetilde{L}$, we know that $l_{\rho}$ is distinct from all the preceding $l_{\nu}$ 's.

The case where $\rho=\mu+1$ is a successor ordinal can be treated like that of a limit ordinal, the only discrepancy being that $\tau_{\rho}=\tau_{\mu+1}$ is already present in the successor case. 
By transfinite induction we thus obtain elements $\tau_{\nu}, x_{\nu}, l_{\nu}$ with the properties (1)-(4) for all $\nu<\Omega$, and our construction permits the formation of the infinite sum $x=\sum_{\nu<\Omega} x_{\nu} \in \prod_{\tau<\Omega} A_{\tau}$. We will reach a contradiction to $f(x) \in \widehat{\prod}_{l \in L} B_{l}$ by showing that $p_{l_{\nu}} f(x) \neq 0$ for all $\nu<\Omega$. In fact, we have

$$
p_{l_{\nu}} f(x)=p_{l_{\nu}} f\left(\sum_{\mu<\nu} x_{\mu}\right)+p_{l_{\nu}} f\left(x_{\nu}\right)+p_{l_{\nu}} f\left(\sum_{\mu>\nu} x_{\mu}\right),
$$

where

$p_{l_{\nu}}\left(\sum_{\mu<\nu} x_{\mu}\right)=0, \quad p_{l_{\nu}} f\left(x_{\nu}\right) \notin F_{\tau_{\nu+1}} B_{l_{\nu}}, \quad$ and $\quad p_{l_{\nu}}\left(\sum_{\mu>\nu} x_{\mu}\right) \in F_{\tau_{\nu+1}} B_{l_{\nu}}$

(the latter holds because $\sum_{\mu>\nu} x_{\mu} \in F_{\tau_{\nu+1}}\left(\prod_{\tau<\Omega} A_{\tau}\right)$ ). This completes the proof of the lemma.

Proof of Theorem 1. We only have to establish that (1) implies (2) and (3) and, as indicated above, it suffices to show this for the case where $J^{2}=0$ and $J / J^{2}$ has $R / J$-dimension 2 . It is thus enough to prove that, for $M=\bigoplus_{\alpha \in k} M_{\alpha}$ and any filter $\mathscr{F}$ on $\mathbb{N}$, the reduced power $M^{\mathbb{N}} / \mathscr{F}$ fails to be algebraically compact. Preserve the complete notational set-up preceding Lemmas 3 and 4.

Let $\mathscr{F}$ be given, and assume that $M^{\mathbb{N}} / \mathscr{F}$ is algebraically compact. Since the inclusion $M \cong \Delta(M) \subseteq M^{\mathbb{N}} / \mathscr{F}$ is pure by Lemma 4 , the reduced power $M^{\mathbb{N}} / \mathscr{F}$ contains a pure-injective envelope $\bar{E}$ of $\Delta(M)$. Using Lemma 4 again, we observe that $\Delta(M)$ is pure in $(\bar{E}+\operatorname{Ker}(\Phi)) / \operatorname{Ker}(\Phi) \cong \bar{E} /(\bar{E} \cap \operatorname{Ker}(\Phi))$, and therefore $\bar{E} \cap \operatorname{Ker}(\Phi)=0$. Consequently, $\Phi$ maps $\bar{E}$ onto an isomorphic copy $\widetilde{E}$ which is contained in $\widehat{\prod}_{\alpha \in k} U_{\alpha}$, where $U_{\alpha}=M_{\alpha}^{\mathbb{N}} / \mathscr{F}$. In view of Lemma $2, \widetilde{E}$ is isomorphic to $\prod_{\alpha \in k} M_{\alpha}$, which yields an embedding

$$
f: \prod_{\alpha \in k} M_{\alpha} \rightarrow \widehat{\prod_{\alpha \in k}} U_{\alpha}
$$

We will prove this to be impossible by showing that the full direct product $\prod_{\alpha \in k} M_{\alpha}$ is "algebraically too thick" to fit into the "thinned product" $\hat{\prod}_{\alpha \in k} U_{\alpha}$. In order to make Lemma 5 applicable, consider a well-ordering of $k$, say

$$
k=\left\{\alpha_{\tau} \mid \tau<\Omega\right\}
$$


where $\Omega$ again stands for the first uncountable ordinal number, and introduce the functors

$$
F_{\tau}: \operatorname{Mod}-R \rightarrow \operatorname{Mod}-\mathbb{Z}, \quad A \rightarrow \bigcap_{\nu \leq \tau}\left(A\left(x-y \alpha_{\nu}\right)\right)
$$

for $\tau<\Omega$. Note that these functors satisfy the prerequisites for an application of Lemma 5 , whence the latter yields an ordinal number $\rho<\Omega$, an uncountable subset $\widetilde{k}$ of $k$, and a countable subset $\widetilde{L}$ of $k$ such that

$$
f\left(F_{\rho} \prod_{\alpha \in \widetilde{k}} M_{\alpha}\right) \subseteq \prod_{l \in \widetilde{L}} U_{l}+\bigcap_{\tau<\Omega} F_{\tau}\left(\widehat{\prod} U_{\alpha \in k}\right) .
$$

Next observe that

$$
F_{\tau} M_{\alpha_{\sigma}}= \begin{cases}0 & \text { for } \sigma \leq \tau, \\ M_{\alpha_{\sigma}} J & \text { for } \sigma>\tau,\end{cases}
$$

where $J=(x, y)$ is the Jacobson radical of $R$, and deduce that the intersection on the right-hand side of the above inclusion vanishes.

Since $\widetilde{L} \cup\left\{\alpha_{\tau} \mid \tau \leq \rho\right\}$ is a countable subset of $k$ and $\widetilde{k} \subseteq k$ is uncountable, there exists moreover an element $\gamma \in \widetilde{k}, \gamma \notin \widetilde{L} \cup\left\{\alpha_{\tau} \mid \tau \leq \rho\right\}$. In particular, our construction thus yields

$$
M_{\gamma} J \cong f\left(M_{\gamma} J\right)=f\left(F_{\rho} M_{\gamma}\right) \subseteq \prod_{l \in \widetilde{L}} U_{l}
$$

Write $f\left(M_{\gamma}\right)$ in the form $z R$ with $z=\left(z_{\alpha}\right)_{\alpha \in k} \in \widehat{\prod}_{\alpha \in k} U_{\alpha}$, and verify the following annihilator computation:

$$
\operatorname{Ann}(z)=(x-y \gamma)=\bigcap_{\alpha \in k} \operatorname{Ann}\left(z_{\alpha}\right)=\operatorname{Ann}\left(z_{\gamma}\right) .
$$

(While the first two equalities are obvious, the last follows from the fact that each $\operatorname{Ann}\left(z_{\alpha}\right)$ is one of $(x-y \alpha)$ or $J$ or $R$ and that $(x-y \alpha) \cap(x-y \gamma)=0$ for $\alpha \neq \gamma$, because $\alpha \not \equiv \gamma(\bmod J)$.) This entails $\operatorname{Ann}\left(z_{\gamma}\right) \subsetneq J$, whereas, on the other hand, $z J=f\left(M_{\gamma} J\right) \subseteq$ $\prod_{l \in \tilde{L}} U_{l}$ shows $z_{\gamma} J=0$ (because $\gamma \notin \widetilde{L}$ ), a contradiction.

3. Ultrapowers and pure global dimension of group algebras. With the aid of a few harmless reduction steps we can extend the "commutative results" of the preceding section to group algebras. Given an uncountable field $K$ of positive characteristic $p$ and a finite group $G$, let $K G$ be the corresponding group algebra. "Module" will mean unitary right module. 
THEOREM 6. The following statements are equivalent:

(1) $K G$ is not of finite representation type.

(2) There exists an $\aleph_{1}$-generated $K G$-module $M$ such that, for each filter $\mathscr{F}$ on $\mathbb{N}$, the reduced product $M^{\mathbb{N}} / \mathscr{F}$ fails to be algebraically compact.

The proof will be based on the following two lemmas.

LEMmA 7. Let $S \subseteq R$ be a ring extension such that $R$ is finitely generated and free as a left $S$-module and contains $S$ as an $S-S$ bimodule direct summand. Then the following are true:

(a) The functor $-\otimes_{S} R:$ Mod $-S \rightarrow$ Mod $-R$ commutes with arbitrary reduced products (modulo filters).

(b) Let $M$ be a right $S$-module. If the $R$-module $M \otimes_{S} R$ is algebraically compact, then the same is true for the $S$-module $M$.

Proof. Part (a) is trivial. For part (b) observe that if $M \otimes_{S} R$ is algebraically compact as an $R$-module, then $M \otimes_{S} R$ is a fortiori algebraically compact as an $S$-module. Moreover, the hypothesis forces $M$ to be a direct summand of the latter $S$-module.

LeMMA 8 (well-known). A finite $p$-group $P$ is either cyclic or contains a normal subgroup $N$ such that $P / N \cong \mathbb{Z} /(p) \times \mathbb{Z} /(p)$.

Proof of Theorem 6. “(2) $\Rightarrow(1)$ " is clear by the remarks in the introduction.

Assume (1), and let $P$ stand for a Sylow $p$-subgroup of $G$. Since $K G$ is a finitely generated and free left $K P$-module, Lemma 7 shows that it suffices to find a $K P$-module $M$ with the properties under (2). The group $P$ being non-cyclic by [9], Lemma 8 provides us with a normal subgroup $N$ of $P$ such that $P / N \cong \mathbb{Z} /(p) \times \mathbb{Z} /(p)$. Next observe that $K(P / N) \cong K P / \omega(N)$ where $\omega(N)$ is the ideal of $K P$ generated by the augmentation ideal of $K N$. It is therefore enough to secure an $\aleph_{1}$-generated module $M$ over the ring $K(\mathbb{Z} /(p) \times \mathbb{Z} /(p))$ with $M^{\mathbb{N}} / \mathscr{F}$ failing to be algebraically compact over the latter ring. But, since this ring is commutative and not of finite representation type [7], Theorem 1 yields such a module $M$.

4. Appendix. Some "essentially" known algebraic compactness results. Here $R$ denotes an arbitrary ring with identity, and " $R$-module" stands for "unitary right $R$-module." 
Given an infinite cardinal number $\aleph$, call an $R$-module $M \aleph$-algebraically compact in case every finitely solvable system of equations

$$
\sum_{i \in I} X_{i} r_{i j}=m_{j} \quad(j \in J),
$$

where $\left(r_{i j}\right)_{i \in I, j \in J}$ is a column-finite $R$-matrix, $m_{j} \in M$ and $|J| \leq \aleph$, has a global solution in $M^{I}$.

Call $M \aleph$-presented if there exists an exact sequence

$$
R^{(\aleph)} \rightarrow R^{(\aleph)} \rightarrow M \rightarrow 0 .
$$

For $\aleph \geq \max \left(|R|, \aleph_{0}\right)$, every $\aleph$-generated $R$-module is clearly $\aleph$-presented. Moreover, again letting $\aleph \geq \max \left(|R|, \aleph_{0}\right)$, every $R$-module is the union of its $\aleph$-presented pure submodules. (In fact, given an $R$ module $M$ and $m \in M$, set $M_{0}=m R$ and construct an ascending sequence $M_{0} \subseteq M_{1} \subseteq M_{2} \subseteq \cdots$ of submodules of $M$ with $\left|M_{n}\right| \leq \aleph$ as follows: Given $M_{n}$, pick a solution $m^{(s)}$ in $M^{I}$ for each finite system $(s)$ which is solvable in $M$ and has the form

$$
\text { (s) } \sum_{i \in I} X_{i} r_{i j}=m_{j} \quad(j \in J)
$$

with $r_{i j} \in R$ and $m_{j} \in M_{n}$. Define $M_{n+1}$ to be the submodule of $M$ generated by $M_{n}$ and the elements of $M$ involved in the $m^{(s)} \in M^{(\mathbb{N})}$. Then $U=\bigcup_{n \in \mathbb{N}} M_{n}$ is a pure submodule of $M$ with $m \in U$ and $|U| \leq \aleph$.)

Using the well-known equivalence of algebraic compactness and pure-injectivity [10, Theorem 2], we will give a direct, very elementary argument for the fact that for any infinite ring $R$ and any $R$-module $M$, algebraic compactness of $M$ follows from $|R|$-algebraic compactness. In fact, in view of the above comments, this is an immediate consequence of the following theorem.

TheOREM A [6, Théorème 7.11]. Suppose that $\aleph$ is an infinite cardinal number such that every $R$-module is the union of its $\aleph$-presented pure submodules. Then every $\aleph$-algebraically compact $R$-module is algebraically compact.

A proof will result from the second of the following two lemmas.

Lemma B. Suppose that $M$ is an $\aleph$-algebraically compact $R$-module and that $A \subseteq B$ is a pure inclusion of $R$-modules such that $B / A$ is 
$\aleph$-presented. Then every map in $\operatorname{Hom}_{R}(A, M)$ can be extended to one in $\operatorname{Hom}_{R}(B, M)$.

Proof. Let $f \in \operatorname{Hom}_{R}(A, M)$. Pick $b_{i} \in B, i \in I$, such that $\left(\overline{b_{i}}\right)_{i \in I}$ is a generating system of $B / A$; moreover, let $\left(r_{j}\right)_{j \in J}$ with $r_{j} \in R^{(I)}$ be a generating system for the relations among the $\bar{b}_{i}$ such that $|J| \leq \aleph$. Setting $a_{j}=\sum_{i \in I} b_{i} r_{i j}$, where $\left(r_{i j}\right)_{i \in I}=r_{j}$, we obtain $a_{j} \in A$, and the purity of $A \subseteq B$ guarantees finite solvability in $A$ of the following system of equations:

$$
\sum_{i \in I} X_{i} r_{i j}=a_{j} \quad(j \in J) .
$$

Consequently, the system

$$
\sum_{i \in I} X_{i} r_{i j}=f\left(a_{j}\right) \quad(j \in J)
$$

is finitely solvable in $M$, and hence is globally solvable by hypothesis. If $\left(m_{i}\right)_{i \in I} \in M^{I}$ is a solution, then there is a well-defined map $g \in$ $\operatorname{Hom}_{R}(B, M)$ with $\left.g\right|_{A}=f$ and $g\left(b_{i}\right)=m_{i}$.

Lemma C. Suppose that $M$ is $\aleph$-algebraically compact and that $A \subseteq$ $B$ is a pure inclusion of $R$-modules such that every quotient module of $B / A$ is the union of its $\aleph$-presented pure submodules. Then every homomorphism $A \rightarrow M$ can be extended to a homomorphism $B \rightarrow M$.

Proof. Given $f \in \operatorname{Hom}_{R}(A, M)$, we will use a transfinite induction to construct, for each ordinal number $\alpha$, an $R$-module $E_{\alpha}$ between $A$ and $B$ and a map $f_{\alpha} \in \operatorname{Hom}_{R}\left(E_{\alpha}, M\right)$ extending $f$ such that

(1) $E_{0}=M, f_{0}=f$.

(2) Each $E_{\alpha}$ is pure in $B$.

(3) $E_{\alpha} \varsubsetneqq E_{\beta}$ whenever $E_{\alpha} \neq B$ and $\alpha<\beta$.

(4) $\left.f_{\beta}\right|_{E_{\alpha}}=f_{\alpha}$ whenever $\alpha<\beta$.

This construction will prove the lemma, because for every ordinal $\alpha$ with $\alpha>|M|$ we will have $E_{\alpha}=B$.

Suppose we already have $E_{\alpha}$ and $f_{\alpha}$ as desired for all $\alpha<\gamma$, where $\gamma \geq 1$.

In case $\gamma$ is a limit ordinal, the union $E_{\gamma}=\bigcup_{\alpha<\gamma} E_{\alpha}$, together with the obvious "patchwork" extension of the $f_{\alpha}$ to $f_{\gamma} \in \operatorname{Hom}_{R}\left(E_{\gamma}, M\right)$, is as desired. 
Now suppose $\gamma=\beta+1$. In case $E_{\beta}=B$, set $E_{\gamma}=E_{\beta}$ and $f_{\gamma}=f_{\beta}$. If $E_{\beta} \varsubsetneqq B$, our hypothesis guarantees the existence of a non-zero, pure, א-presented submodule $U$ of $B / E_{\beta} \cong(B / A) /\left(E_{\beta} / A\right)$. Let $E_{\gamma} \subseteq B$ be the preimage of $U$ under the canonical epimorphism $B \rightarrow B / E_{\beta}$. Then $E_{\gamma}$ is pure in $B$ (because the inclusions $E_{\beta} \subseteq B$ and $E_{\gamma} / E_{\beta} \subseteq B / E_{\beta}$ are both pure), $E_{\beta} \varsubsetneqq E_{\gamma}$, and $E_{\gamma} / E_{\beta}$ is $\aleph$-presented. By Lemma B we can thus extend $f_{\beta}$ to $f_{\gamma} \in \operatorname{Hom}_{R}\left(E_{\gamma}, M\right)$.

THEOREM D (compare [4, Theorem 42.1] and [6, Remarque 9.12]). Let $\left(M_{i}\right)_{i \in I}$ be any family of $R$-modules, and let $\mathscr{F}$ be a filter on I which contains a countable chain

$$
F_{1} \supseteq F_{2} \supseteq F_{3} \supseteq \cdots
$$

with empty intersection. Then the reduced product $\prod_{i \in I} M_{i} / \mathscr{F}$ is $\aleph_{0}$ algebraically compact.

CoROllaRy E. If $|R| \leq \aleph_{0}$, then every reduced product $\prod_{n \in \mathbb{N}} M_{n} / \mathscr{F}$ of $R$-modules, where $\mathscr{F}$ is a filter containing all cofinite subsets of $\mathbb{N}$, is algebraically compact. In particular, each non-trivial countable ultraproduct of $R$-modules is algebraically compact.

Proof of Corollary E. Combine Theorems A and D.

Proof of Theorem D. Set $M=\prod_{i \in I} M_{i} / \mathscr{F}$, and consider a countable, finitely solvable system of equations:

$$
\sum_{l \in L} X_{l} r_{l j}=m_{j} \quad(j \in \mathbb{N})
$$

with $m_{j}=\left[m_{j i}\right]_{i \in l} \in M$. For each $k \in \mathbb{N}$ pick a solution $\left(a_{l}^{(k)}\right)_{l \in L} \in M^{L}$ of the first $k$ equations, i.e.,

$$
\sum_{l \in L} a_{l}^{(k)} r_{l j}=m_{j} \quad \text { for } 1 \leq j \leq k .
$$

If we write $a_{l}^{(k)}=\left[a_{l i}^{(k)}\right]_{i \in I} \in M$, the latter equations amount to the existence of a set $\widetilde{F}_{k} \in \mathscr{F}$ with $\widetilde{F}_{k} \subseteq F_{k}$ such that for all $i \in \widetilde{F}_{k}$ we have

$$
\sum_{l \in L} a_{l i}^{(k)} r_{l j}=m_{j i} \quad(1 \leq j \leq k)
$$


Set $F_{-1}=I$, and for each $i \in I$ let $q(i) \geq-1$ be maximal with respect to $i \in F_{q(i)}$. If we define $a_{l i}:=a_{l i}^{(q(i))} \in M_{i}$ for each $i \in I$, then $\left(\left[a_{l i}\right]_{i \in I}\right)_{l \in L} \in M^{L}$ is clearly a global solution of the initial system.

THEOREM F. If $R$ is an Artin algebra and $M$ a finitely generated $R$ module, then, given any index set $I$ and any filter $\mathscr{F}$ on $I$, the reduced power $M^{I} / \mathscr{F}$ is algebraically compact. In particular, all ultrapowers of $M$ are algebraically compact.

Proof. By [14, Proposition 3], $M$ is $\Sigma$-algebraically compact, and so is $M^{I}$. Since $\Sigma$-algebraic compactness is inherited by pure submodules [12, Folgerung 3.4], the submodule

$$
U(\mathscr{F})=\left\{m \in M^{I} \mid I \backslash \operatorname{supp}(m) \in \mathscr{F}\right\}
$$

is a direct summand of $M^{I}$, whence $M^{I} / \mathscr{F}=M^{I} / U(\mathscr{F})$ is in turn $\Sigma$-algebraically compact.

\section{REFERENCES}

[1] M. Auslander, Large modules over Artin algebras, Algebra, Topology, and Category Theory (a collection of papers in honor of S. Eilenberg), pp. 1-17, Academic Press, New York, 1976.

[2] S. U. Chase, Direct products of modules, Trans. Amer. Math. Soc., 97 (1960), 457-473.

[3] On direct sums and products of modules, Pacific J. Math., 12 (1962), 847-854.

[4] L. Fuchs, Infinite Abelian Groups I, II, Academic Press, New York, 1970/1973.

[5] L. Gruson and C. U. Jensen, Deux applications de la notion de L-dimension, C. R. Acad. Sci. Paris, Sér. A 282 (1976), 23-24.

[6] Dimension cohomologique reliées aux foncteurs $\lim ^{(l)}$, in: Séminaire d'Algèbre Paul Dubreil et Marie-Paule Malliavin, Lecture Notes in Mathematics No. 876, 1981.

[7] D. G. Higman, Indecomposable representations at characteristic p, Duke Math. J., 21 (1954), 377-381.

[8] C. U. Jensen and H. Lenzing, Algebraic compactness of reduced products and applications to pure global dimension, Comm. Algebra, 11 (1983), 305-325.

[9] F. Kasch, M. Kneser and H. Kupisch, Unzerlegbare modulare Darstellungen endlicher Gruppen mit zyklischer p-Sylowgruppe, Arch. Math., 8 (1957), 320321.

[10] R. B. Warfield, Jr., Purity and algebraic compactness for modules, Pacific J. Math., 28 (1969), 699-719.

[11] Large modules over artinian rings, Representation theory of algebras (Proc. Conf., Temple Univ., Philadelphia, Pa., 1976), pp. 451-463, Lecture Notes in Pure Appl. Math. 37, Dekker, New York, 1978. 
[12] W. Zimmermann, Rein injektive direkte Summen von Moduln, Comm. Algebra, 5 (1977), 1083-1117.

[13] B. Zimmermann-Huisgen, Rings whose right modules are direct sums of indecomposable modules, Proc. Amer. Math. Soc., 77 (1979), 191-197.

[14] B. Zimmermann-Huisgen and W. Zimmermann, Algebraically compact rings and modules, Math. Z., 161 (1978), 8i-93.

Received February 23, 1988.

Københavns Universitets Matematiske Institut

UNIVERSITETSPARKEN 5

2100 KøbEnHAVN $\varnothing$, DanmaRK

AND

UNIVERSITY OF CALIFORNIA

SANTA BARbara, CA 93106, USA 
